\title{
APLICAÇÃO DA ESCALA DE FRAMINGHAM NA UNIDADE BASICA DE SAÚDE DO BAIRRO HORTO FLORESTAL DO MUNICÍPIO DE ITAPERUNA-RJ
}

\author{
Wilian de Freitas JUNIOR*, Gisela Machado ALTOÉ, Nikyallan Soares RODRIGUES, Thays Costa \\ GRAVINA, Karoline FERNANDES \& Juçara Golçalves Lima BEDIM
}

Universidade Iguaçu, campus V. Itaperuna, Rio de Janeiro, Brasil.

*Autor para correspondência: wilianjrfreitas@ hotmail.com

DOI: http://dx.doi.org/10.18571/acbm.127

\section{RESUMO}

Há consenso sobre a importância da adoção de estratégias de atenção integral à saúde focadas na prevenção do aparecimento de comorbidades, dentre inúmeros fatores, tais como: envelhecimento da população, sobrevida das doenças infecciosas, sendo que uma parcela importante pode ser atribuída ao controle inadequado dos fatores associados ao desenvolvimento dessas doenças. $\mathrm{O}$ presente estudo teve como objetivo analisar e avaliar o perfil dos pacientes acompanhados em uma Unidade Básica de Saúde (UBS), com a perspectiva de prevenção de sofrimento de um evento coronariano agudo, contribuindo para a melhoria da qualidade de vida dos atores sociais do Noroeste Fluminense. O universo da pesquisa foi a UBS do bairro Horto Florestal, Itaperuna/RJ, onde foi realizado um estudo analítico observacional transversal compreendendo a aplicação da Escala de Framingham. Os critérios de inclusão foram pacientes em idade entre 30 e 74 anos, de forma randomizada, no período de agosto/2014, abri1/2015. Dentre os preditores ressaltam-se LDL colesterol, HDL colesterol, pressão arterial sistêmica, diabetes mellitus, tabagismo. Faz-se mister elucidar os resultados mais significativos: $44,8 \%$ dos indivíduos pontuaram risco através do HDL colesterol; quanto aos níveis de pressão arterial, mais de $50 \%$ somaram pontos no escore. Referindo-se ao diabetes, $16,2 \%$ apresentaram a doença e ao tabagismo, $20 \%$, sendo que estes dois últimos agravam ainda mais o risco de evento cardiovascular. Estudos embasados na integração com a rede de serviços de saúde contribuem para a prevenção de agravos, constituindo-se estratégias significativas para a construção de um país mais justo, onde a saúde promove cidadania na sua integralidade.

Palavras-chave: Rede de Saúde; Escala de Framingham; Risco; Prevenção.

\begin{abstract}
There is consensus about the importance of adopting comprehensive health care strategies focused on preventing the onset of comorbidities, among innumerable factors, such as: aging of the population, survival of infectious diseases, and an important portion can be attributed to inadequate control of Factors associated with the development of these diseases. The present study aimed at analyzing and evaluating the profile of patients followed at a Basic Health Unit (BHU), with the perspective of preventing the suffering of an acute coronary event, contributing to the improvement of the quality of life of the social actors of the Northwest Fluminense. The research universe was the UBS of the Horto Florestal neighborhood, Itaperuna / RJ, where an observational cross-sectional analytical study was carried out, including the Framingham scale. Inclusion criteria were patients aged between 30 and 74 years, being randomly, in the period of August / 2014, April / 2015. Among the predictors are: LDL cholesterol, HDL cholesterol, systemic arterial pressure, diabetes mellitus, smoking. It is necessary to elucidate the most significant results: $44.8 \%$ of the individuals scored at risk through HDL cholesterol; As for blood pressure levels, more than 50\% scored points in the score. Referring to diabetes, $16.2 \%$ had the disease and smoking, $20 \%$, the latter two worsening the risk of cardiovascular events. Studies based on integration with the health
\end{abstract}


services network contribute to the prevention of diseases and constitute significant strategies for building a more just country, where health promotes citizenship in its entirety.

Keywords: Health Network; Framingham scale; Risk; Prevention.

\section{Introdução}

No ano de 1948, foi iniciado nos EUA um estudo de coorte para compreender os fatores de risco associados às doenças cardiovasculares. Esse estudo foi iniciado numa época em que a mortalidade e a incidência da Doença Cardiovascular (DCV) vinham aumentando progressivamente e pouco se conhecia acerca dos fatores de risco e fisiopatologia (OLIVEIRA et al., 2007).

A cidade de Framingham, Massachussets, foi escolhida para sediar o estudo. Foram recrutadas 5209 pessoas com idades entre 30 e 62 anos e, em seguida, foram realizados cuidadosos exames físicos e extensas anamneses, tendo atenção especial aos hábitos de vida, para que se pudesse analisar os padrões comuns relacionados ao desenvolvimento de DCV. A partir de então, essas pessoas são reavaliadas de dois em dois anos e, até hoje, esse estudo vem sendo feito, sendo incorporadas as novas gerações desta população. Toda essa monitorização levou à identificação dos principais fatores de risco de DCV, como hipertensão arterial sistêmica, dislipidemia, tabagismo, obesidade, diabetes e sedentarismo, bem como uma grande quantidade de informações valiosas sobre os efeitos de fatores relacionados, tais como os níveis de colesterol HDL e triglicerídeos, idade, gênero e questões psicossociais (FRAMINGHAM, 2008).

A partir daí, foi criada uma escala na qual é possível prever a ocorrência de DCV em 10 anos num indivíduo, utilizando informações como idade, sexo, LDL, HDL, tabagismo, hipertensão arterial e diabetes mellitus. A pontuação varia de $<-3$ a $>14$, o que representa risco de respectivamente de $1 \%$ e $>55 \%$ (BRASIL, 2006). Contudo, essa escala não é isenta de falhas. $\mathrm{O}$ estudo de Framingham, que deu origem a esse escore, foi realizado apenas numa parcela da população do nordeste americano, portanto, seus resultados não levam em consideração as diferenças étnicas. Além disso, vários fatores não foram tão valorizados nessa metodologia, como o Diabetes Mellitus e menopausa precoce (MAFRA et al., 2008).

Diante de tais fatores, um outro estudo vem sendo realizado desde 1994, com a perspectiva de suprir as falhas do estudo anterior, mas, ainda, não chegando ao ideal. Trata-se do estudo de SCORE, elaborado por várias sociedades europeias, reunindo mais de 25.000 pessoas de diversos países do continente que são acompanhadas por dez anos. Esse estudo leva em conta apenas o risco de evento coronariano fatal, não prevendo a totalidade de eventos cardiovasculares. Da mesma maneira que o estudo de Framingham, o de SCORE, igualmente, apresenta algumas falhas por não englobar todos os fatores predisponentes ao evento coronariano, utilizando apenas como variáveis gênero, idade, tabagismo, colesterol total e pressão arterial sistólica.

Após os estudos supracitados, diversos outros foram publicados com a finalidade de compreender o impacto da eliminação de fatores que contribuem para a incidência de DCV. Um deles é o Multiple Risk Factor Intervention Trial (MRFIT), realizado entre 1972 e 1973, nos Estados Unidos. Tratou-se de um estudo randomizado em que três dos maiores fatores de risco tabagismo, hipercolesterolemia e hipertensão arterial - foram eliminados, com o objetivo de observar o impacto sobre a mortalidade por doença coronariana (MELO FILHO, 1998; POZZAN et al., 2004).

Nesse contexto, o escore de Framingham ainda continua sendo o mais utilizado atualmente para quantificar o risco de evolução para eventos cardiovasculares pelos pacientes.

Desde a implantação do Sistema Único de Saúde (SUS), no Brasil, a estratégia de atendimento à população dá-se através da divisão da assistência em três esferas, sendo a esfera Primária/Básica - Atendimento Primário - a porta de entrada da população aos serviços de saúde 
e, além das atividades curativas, as atividades preventivas ganharam importante destaque na assistência à população.

A Saúde da Família é entendida como uma estratégia de reorientação do modelo assistencial, operacionalizada mediante a implantação de equipes multiprofissionais em Unidades Básicas de Saúde (UBS). Estas equipes são responsáveis pelo acompanhamento de um número definido de famílias, localizadas em uma área geográfica delimitada. As equipes atuam com ações de promoção à saúde, prevenção, recuperação, reabilitação de doenças e agravos mais frequentes, e na manutenção da saúde desta comunidade.

Cada equipe se responsabiliza pelo acompanhamento de cerca de 3 mil a 4 mil e 500 pessoas ou de mil famílias de uma determinada área, e estas passam a ter co-responsabilidade no cuidado à saúde. A atuação das equipes ocorre principalmente nas UBS, nas residências e na mobilização da comunidade, caracterizando-se: como porta de entrada de um sistema hierarquizado e regionalizado de saúde; por ter território definido, com uma população delimitada, sob a sua responsabilidade; por intervir sobre os fatores de risco aos quais a comunidade está exposta; por prestar assistência integral, permanente e de qualidade; por realizar atividades de educação e promoção à saúde e, ainda: por estabelecer vínculos de compromisso e de coresponsabilidade com a população; por estimular a organização das comunidades para exercer o controle social das ações e serviços de saúde; por utilizar sistemas de informação para o monitoramento e a tomada de decisões; por atuar de forma intersetorial, por meio de parcerias estabelecidas com diferentes segmentos sociais e institucionais, de forma a intervir em situações que transcendem a especificidade do setor saúde e que têm efeitos determinantes sobre as condições de vida e saúde dos indivíduos-famílias-comunidade (LEI 8080/1990). Desse modo, representa um dos pilares do modelo assistencial à saúde no Brasil, consistindo na prevenção de agravos.

Há consenso sobre a importância da adoção de estratégias de atenção integral, cada vez mais precoces ao longo do ciclo de vida, focadas na prevenção do aparecimento dessas comorbidades. O principal desafio é traduzir em ações concretas de cuidado integral a indivíduos e comunidades o conhecimento científico e os avanços tecnológicos, hoje, disponíveis e colocálos em prática ao alcance de um maior número possível de indivíduos.

Por conseguinte, na trajetória dos pressupostos elucidados acima, este estudo teve por objetivo investigar fatores que mais contribuem para o aumento do risco de desenvolvimento de doenças cardiovasculares nos pacientes acompanhados na UBS Horto Florestal, Itaperuna-RJ, com vistas a intervir nos hábitos de vida desses sujeitos-atores, e diminuir o risco de sofrimento de um evento coronariano agudo. Consequentemente, contribuir para a melhora da qualidade de vida dos atores sociais.

As doenças circulatórias são responsáveis por impacto expressivo na mortalidade da população brasileira. São inúmeros os fatores que podem estar relacionados com a importância cada vez maior dessas doenças, dentre eles destacando-se envelhecimento da população, sobrevida das doenças infecciosas, incorporação de novas tecnologias com diagnóstico mais precoce das doenças e redução de letalidade, mas uma parcela importante pode ser atribuída ao controle inadequado, e por vezes em ascensão, dos fatores associados ao desenvolvimento de tais doenças (BRASIL, 2006).

Justamente, esses fatores que desencadeiam as síndromes coronarianas agudas e seu impacto expressivo nas questões de saúde ensejaram a presente pesquisa, de forma a intervir de forma colaborativa na prevenção das referidas doenças; donde se aponta a relevância do estudo e se justifica sua realização.

De fato, segundo o Ministério da Saúde (BRASIL, 2006):

Existem inúmeras intervenções protetoras vasculares de benefício comprovado, que visam uma melhora das doenças cardiovasculares. Entre elas destacam-se adoção de hábitos alimentares adequados e saudáveis, cessação do tabagismo, prática de atividade 
física regular, controle da pressão arterial, manejo das dislipidemias, manejo do diabete com controle da glicemia e uso profilático de alguns fármacos.

2006):
Algumas dessas possíveis intervenções: carecem de ser mencionadas, tais como (BRASIL,

1) “Alimentação saudável”, ou seja, reduzir sal e temperos prontos na cozinha, evitar comidas industrializadas e lanches rápidos; limitar a ingestão de açúcar livre, açúcar de mesa, refrigerantes e sucos artificiais, doces e guloseimas em geral; ingerir frutas, legumes e verduras diariamente; aumentar consumo de cereais integrais e leguminosas; reduzir o consumo de carnes gordurosas, embutidos, leite e derivados integrais. Igualmente, preferir óleos vegetais como soja, canola, oliva; retirar a gordura aparente de carnes, pele de frango e couro de peixe antes do preparo; incentivar o consumo de peixes e evitar o consumo em excesso de álcool.

2) "Controle do peso", diagnosticado através do Índice de Massa Corporal (IMC); indivíduos com IMC entre 25 e $29,9 \mathrm{~kg} / \mathrm{m}^{2}$ são considerados pré-obesos e se associados com obesidade central ou outros fatores de risco, devem ter intervenção e ser acompanhados. Em indivíduos acima do peso ideal, a redução de peso minimiza o risco cardiovascular; a curto e médio prazo reduz os níveis de glicemia, pressão arterial e melhora o perfil lipídico. Desse modo é importante a mudança dos hábitos de vida, visando à redução do peso com praticas alimentares e atividade física regular.

3) "Atividade física" a qual, se feita regularmente, promove o efeito protetor para doenças cardiovasculares, sendo que a recomendação é que seja realizada por, pelo menos, 30 minutos em intensidade moderada no mínimo três vezes por semana. É importante, também, associar essas atividades à pratica do dia a dia, como subir escadas, realizar atividades domésticas etc.

4) "Tabagismo", recomendando-se o abandono efetivo do hábito/addiction, que pode ser por aconselhamento, ou farmacológico (terapia de reposição da nicotina, encontradas em adesivos e goma de mascar, além de antidepressivos e anti-hipertensivos) que teria um papel eficaz na redução dos sintomas de abstinência.

5) "Farmacoterapia": uso de anti-hipertensivos (tiazídicos e IECA) que visam redução dos níveis pressóricos, uso de antiplaquetários em especial aspirina em dose baixa (100mg/dia); fármacos hipolipemiantes (estatinas) que buscam reduzir os níveis séricos de colesterol e, consequentemente, os eventos vasculares.

\section{Material e Métodos}

Trata-se de um estudo analítico observacional transversal, cujo universo da pesquisa constituiu-se de pacientes acompanhados na UBS Horto Florestal, inserida no Programa de Agentes Comunitários da Saúde (PACS), segmento da Secretaria Municipal de Saúde de Itaperuna-RJ.

Através da coleta sistematizada de informações para a determinação dos escores de Framingham, o mote foi traçar o perfil dos pacientes que apresentam algum risco de desenvolver doença cardiovascular aguda.

Os critérios de inclusão foram pacientes que se encontravam em acompanhamento na UBS e que tinham idade entre 30 e 74 anos, sendo selecionados aproximadamente 100 participantes para a pesquisa, de forma randomizada.

Os preditores utilizados para avaliação foram idade, gênero, LDL colesterol, HDL colesterol, pressão arterial sistêmica, diagnóstico prévio de diabetes mellitus, e tabagismo. Sendo que cada item corresponde a uma pontuação dada pelo escore de Framinghan.

No que se refere ao protocolo para coleta de dados, foi feita a aplicação da escala de Framingham, preenchida a partir das informações relatadas pelos pacientes durante as consultas e 
após o recebimento dos resultados dos exames laboratoriais relativos ao protocolo, juntamente com visita domiciliar para entrevista e aferição da pressão arterial sistêmica. Para isso, foi estabelecida parceria com o Laboratório de Análises Clínicas da Associação Santo Antônio dos Pobres de Itaperuna, e com o médico responsável pelos atendimentos na UBS no período da pesquisa.

Os dados foram analisados com o uso do software Microsoft Excel ${ }^{\circledR}$ (Microsoft Corporation, Washington - USA).

No tocante às considerações éticas, os pacientes envolvidos neste estudo assinaram o "Termos de Consentimento Livre e Esclarecido", autorizando a utilização de suas informações. Com este documento os pesquisadores garantiram a privacidade dos pacientes e de seus dados, considerando que não seriam utilizados iniciais de nomes, números de registros ou qualquer outra forma de identificação dos participantes, assim como não seriam feitos quaisquer procedimentos clínicos nestes pacientes.

\section{Resultados}

Tomando-se como aporte o levantamento dos dados no cenário da UBS Horto Florestal, inicia-se a abordagem analítica dos resultados obtidos e concomitante discussão. Dados esses cuja coleta no campo abrangeu de agosto/2014 a abril/2015.

Primordialmente, mister se faz traçar o perfil dos protagonistas que compuseram o universo amostral da investigação, ou seja, $80 \%$ do sexo feminino e $20 \%$ do sexo masculino, apenas; $20 \%$ na faixa etária até 44 anos; $41 \%$ entre 45 anos e 59 anos; e $39 \%$ acima de 60 anos.

No tocante aos descritores que nortearam a coleta de dados, tais como colesterol, tabagismo, pressão arterial sistêmica e diabetes, segue-se a análise ilustrada pelas figuras originadas.

Segundo Pozzan et al. (2004), níveis elevados de colesterol LDL e níveis baixos de colesterol HDL são dois fatores agravantes na fisiopatologia das doenças cardiovasculares, já que o LDL contribui para a formação da placa aterosclerótica através da agressão ao endotélio vascular e o HDL, quando baixo, deixa de exercer sua função de proteção do leito vascular contra a aterogênese. A partir deste conceito, observamos que uma porcentagem de $64,8 \%$ da população amostral do estudo não pontuou risco dentro do critério LDL (Figura 1), enquanto uma porcentagem de $31,4 \%$ conseguiu reduzir o risco por apresentarem valores séricos dentro da normalidade.

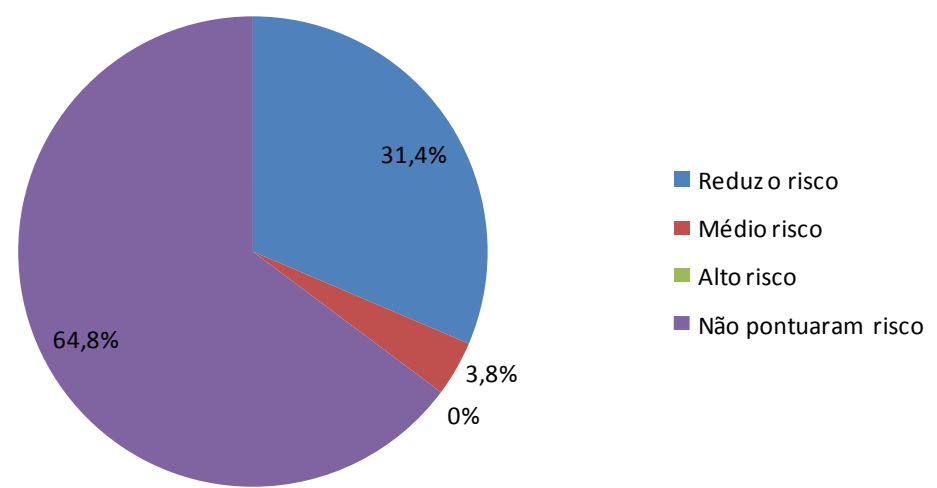

Figura 1: LDL Colesterol. Fonte: Elaboração própria, 2015.

Paralelamente, com relação a figura 2, os valores de colesterol HDL obtidos no grupo amostral do estudo, revelaram que uma parcela mínima da população $(2,9 \%)$ obteve redução no risco cardiovascular, enquanto em $51,8 \%$ o critério não teve relevância na soma final do score e em $45,7 \%$ apresentaram médio $(44,8 \%)$ e alto risco $(0,9 \%)$, com pontuações elevadas no escore, 
demonstrando que apesar de ser um fator redutor na incidência de doenças cardiovasculares quando em níveis aumentados, elevam o risco de surgirem tais doenças.

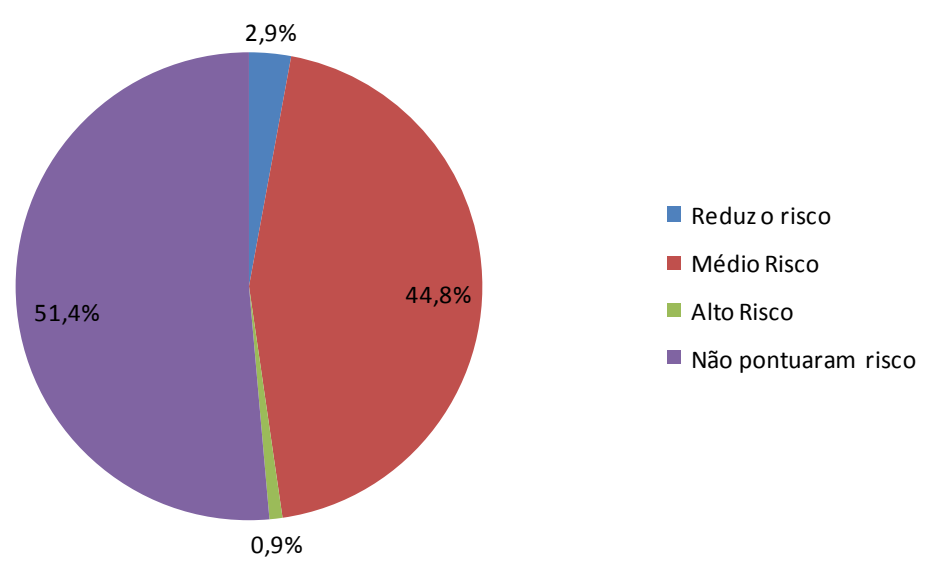

Figura 2: HDL Colesterol. Fonte: Elaboração própria, 2015.

De modo que podemos afirmar que os critérios de colesterol LDL e colesterol HDL não representaram grande relevância no acréscimo de pontos dentro do escore final da maior parcela da população estudada. Acreditamos que tais resultados podem ser atribuídos ao fato de a amostra pesquisada seguir em acompanhamento multidisciplinar na UBS, tendo atitudes que reduzem esses fatores, normalmente mais agravantes na população em geral.

Ao analisarmos a figura 3, percebemos um valor elevado em relação ao médio e alto risco $(29,5 \%$ e $24,8 \%)$ da pressão arterial sistêmica, ou seja, mais da metade dos individuos pesquisados têm uma propensão significativa para desenvolver doenças cardiovasculares. Visto que a maioria das causas de hipertensão está relacionada a predisposições hereditárias e fatores como obesidade, estresse, consumo de álcool e sal, tais condições podem estar contribuindo para hipertensão nesses individuos, sendo um fator agravante na fisiopatologia de doenças cardiovasculares.

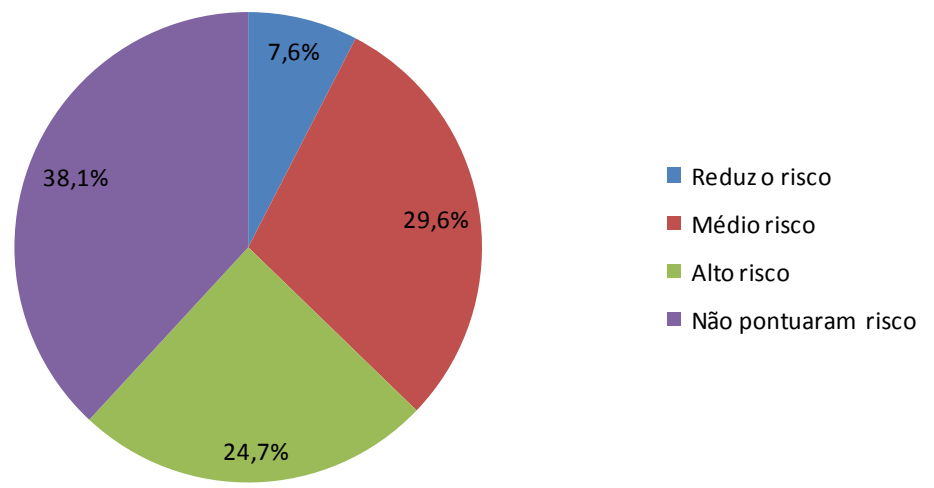

Figura 3: Pressão Arterial Sistêmica. Fonte: Elaboração Própria, 2015.

Conforme elucida Perdigão (2009), os pacientes diabéticos possuem risco aumentado para todos os tipos de doença cardiovascular que culmine ou não com óbito, sendo este risco cardiovascular proporcional ao número de fatores de risco, como tabagismo, hipercolesterolemia e hipertensão arterial, presentes nesses indivíduos. A exploração da figura 4 nos revelou que 88 pessoas do espaço amostral, refletindo o percentual de $83,8 \%$ do total, não possuem diagnóstico de Diabetes Mellitus (DM), enquanto os que padecem da referida síndrome metabólica correspondem a 16,2\%. Apesar da importância do DM, há carência de estudos que investiguem as características epidemiológicas desta condição na população brasileira. Conforme dados encontrados, no final da década de 1980, o Ministério da Saúde, estimou que o diabetes ocorria em cerca de $8 \%$ da população, de 30 a 69 anos de idade, sendo que essa prevalência variava de $3 \%$ 
a 17\% entre as faixas de 30-39 e de 60-69 anos (BRASIL, 2006). Em 2006, segundo o mesmo órgão, estimou-se cerca de 5 milhões e meio de portadores, $11 \%$ da população igual ou superior a 40 anos (conforme população estimada - IBGE, 2005). Desse modo, apesar do resultado encontrado demonstrar estar acima do esperado em âmbito nacional, a comparação é duvidosa e não deve ser feita, pois os estudos contêm viéses quanto à idade.

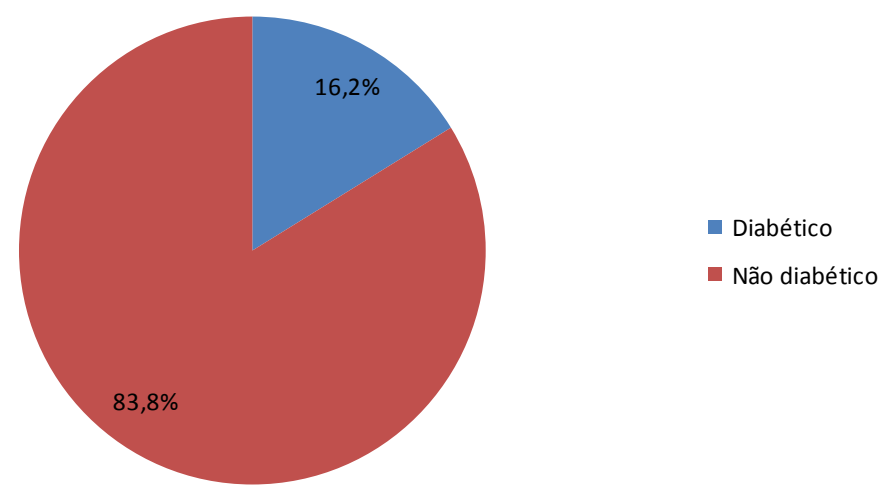

Gráfico 4: Diabetes. Fonte: Elaboração Própria, 2015.

Tendo em vista a importância do tabagismo no contexto de risco cardiovascular, a análise da prevalência do tabagismo na população investigada (Figura 5) demonstrou que 21 indivíduos (20\%) representavam esta parcela. Diante dos resultados, surgiu um questionamento: em base dos critérios de seleção e da faixa etária abordada esperava-se uma porcentagem superior. Ao abordar esses valores à luz dos resultados apresentados pela pesquisa nacional de saúde, 2013, a respeito da prevalência dos indicadores de tabagismo segundo os grupos de idade, observou-se que os resultados analisados em nível local (isto é: $20 \%$ dos participantes) foram, no entanto, superiores aos obtidos em nível nacional nas populações de 25-60 anos ou mais, que variavam de 12,4\%19,4\%. Embora represente ainda uma parcela considerável, dados evidenciados por Monteiro et al. (2007), na primeira pesquisa sobre prevalência de tabagismo no Brasil realizada no ano de 1989, onde estimou-se que $34,8 \%$ da população adulta brasileira era tabagista, demonstram que consumo do tabaco no Brasil declinou nas últimas décadas. Segundo Malta et al. (2015), este declínio deve-se basicamente a ações desenvolvidas nos últimos anos, a exemplo da proibição da propaganda parcial de produtos de tabaco, em 1996, e de legislações subsequentes.

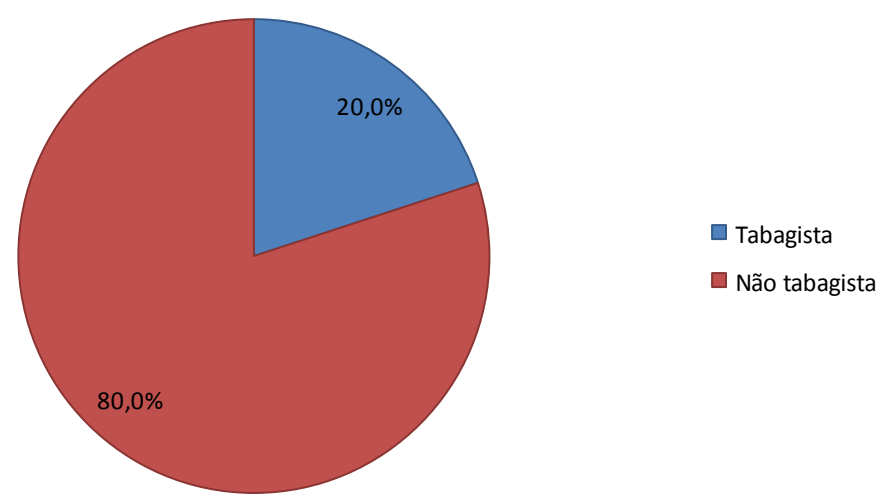

Figura 5: Tabagismo. Fonte: Elaboração Própria, 2015.

\section{Discussão}

Diante dos estudos realizados, primeiramente, o predomínio feminino do universo amostral deixa claro que as mulheres cuidam mais preventivamente da sua saúde, o que segundo Costa Junior e Maia (2009), deve-se ao corpo reprodutivo, enquanto homens cuidam-se menos pois têm 
dificuldades em se afastar do trabalho, procurando por ajuda médica apenas diante de situações críticas. Embora o risco da doença cardiovascular seja semelhante entre homens e mulheres, o que já fora afirmado bem anteriormente por Mosca et al. (1997), o maior acompanhamento médico ambulatorial feminino (pelos pesquisadores deste estudo), dificultou estimar durante a análise se há um gênero com maiores chances de desenvolver a doença cardiovascular na população em estudo. A possibilidade de se estimar o risco absoluto em dez anos permite ações preventivas, principalmente dirigir a estratégia da saúde básica. Dessa forma, ainda se faz necessário suscitar a conscientização da população masculina sobre a importância em se fazer a manutenção da saúde, junto ao corpo multidisciplinar da UBS.

Analisando de forma global a amostra, de acordo com a figura 6, o risco de doença cardiovascular em 10 anos em 79,2\% dos participantes é menor que $15 \%$. E dentre os outros $20,8 \%$, há uma parcela significativa de $4,8 \%$ que tem $27 \%$ de risco cardiovascular na próxima década, o que dentro da avaliação do escore é um alto risco. Os fatores que mais contribuíram para o aumento desse risco foram a hipertensão arterial, o diabetes e o tabagismo, abordados nas figuras 3, 4 e 5 respectivamente. A importância atual está em verificar a presença dos fatores de risco diagnosticados e encorajar os pacientes a tratá-los, incentivando a atividade física, perda de peso e melhora na qualidade de vida, sendo fundamental a estratégia de intervenção da UBS junto a esses cidadãos. Essa atuação, mesmo na presença de um único fator de risco alterado, é fundamental uma vez que a exposição poderá ter desfechos inesperados, fazendo-se necessária a mudança da percepção da população em relação aos fatores de risco para doença cardiovascular.

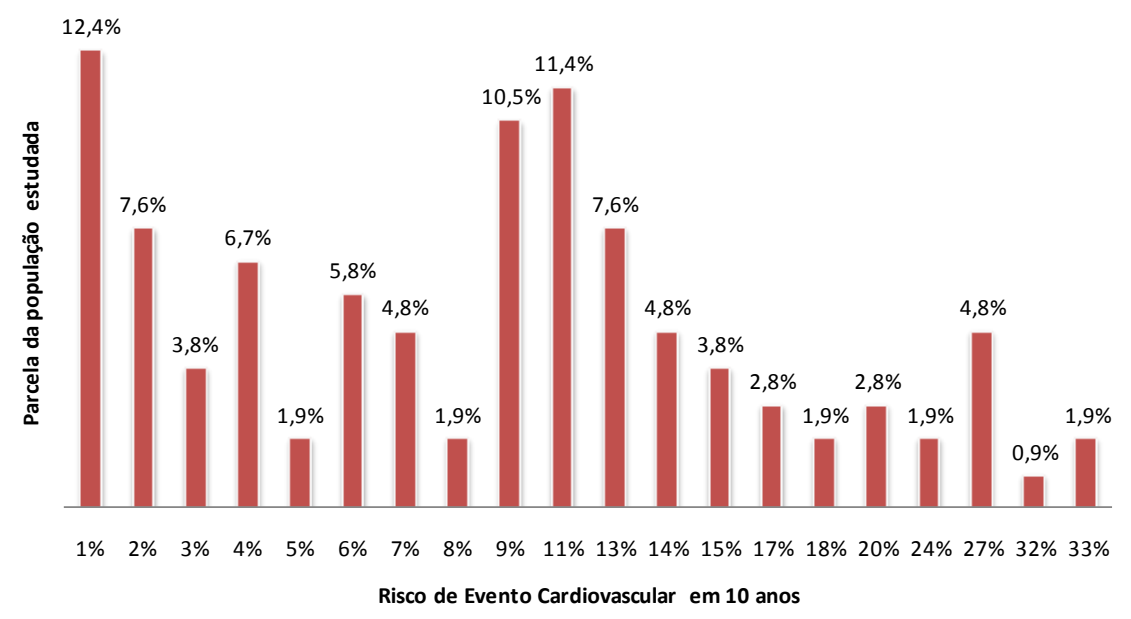

Figura 6: Risco de Evento Cardiovascular em 10 anos. Fonte: Elaboração própria, 2015.

\section{Conclusões}

Em conformidade com a análise de todo material, e o acompanhamento de pacientes na Unidade Básica de Saúde Horto Florestal, no município de Itaperuna-RJ, a pesquisa teve por objetivo traçar o perfil dos pacientes que apresentam risco de desenvolver doença cardiovascular aguda, através do escore de Framingham. Valendo ressaltar, que a melhor maneira de quantificar o risco cardiovascular é considerar todos os fatores envolvidos.

Justifica-se, assim, o interesse de revisitar o assunto, uma vez que fatores desencadeantes de síndromes coronarianas agudas impactam expressivamente nas questões de saúde. Embasando assim uma perspectiva científica atuante no cenário das Estratégias de Saúde da Família, intervindo de forma colaborativa e significativa na prevenção das referidas doenças, onde se apontou a relevância desse estudo.

As evidências acumuladas mediante o escore de risco, apresentam utilidade em práticas de prevenção de doenças cardiovasculares, mas sempre dentro de contexto clínico e epidemiológico. 
Portanto, a promoção, informação, conscientização, e educação continuada são de suma importância.

Conclui-se que, apesar de todos os consensos, o problema ainda é crescente e continua em aberto, ultrapassando o domínio formal do setor saúde, e alcançando o "status" de uma questão pública epistemológica. Espera-se que a pesquisa fomente ideias novas a respeito de mudanças de hábitos, melhora na qualidade de vida e estratégias de intervenção nos fatores abordados pelo escore de Framingham.

\section{Referências}

BRASIL. Ministério da Saúde. Secretaria de Atenção à Saúde. Departamento de Atenção Básica. Diabetes Mellitus. Brasília, 2006.

Ministério da Saúde. Prevenção clínica de doença cardiovascular, cerebrovascular e renal crônica.Cadernos de Atenção Básica, n. 14, Brasília, 2006.

COSTA JÚNIOR, F. M.; MAIA, A. C. B. Psicologia: Teoria e Pesquisa.Concepções de Homens Hospitalizados sobre a Relação entre Gênero e Saúde1 Universidade Estadual Paulista (UNESP), v. 25, n. 1, p. 55-63, 2009.

FRAMINGHAM HEART STUDY, 2008. Disponível em: <www.framinghamheartstudy.org>. Acesso em: 8 abr. 2014.

MAFRA, F.; OLIVEIRA, H. Avaliação do Risco Cardiovascular: metodologias e suas implicações na prática clínica. Revista Portuguesa de Clínica Geral, n. 24, p. 391-400, 2008.

MALTA, et al. Uso e exposição à fumaça do tabaco no Brasil: resultados da Pesquisa Nacional de Saúde 2013. Epidemiol. Serv. Saúde. Brasília, v. 24, n. 2, p. 239-248, abr.jun. 2015.

MELO FILHO, J. X. Multiple Risk Factor Intervention Trial (MRFIT). HiperAtivo, v. 5, n. 1, p. 48-52, 1998.

MONTEIRO, et al. Population-based evidence of a strong decline in the prevalence of smokers in Brazil (1989-2003). Bull World Health Organ, v. 85, n. 7, p. 527-534, jul. 2007.

MOSCA L. et al. Cardiovascular disease in women: a statement for healthcare professionals from the American Heart Association (AHA Scientific Statement). Circulation, n. 96, p. 2468-2482, 1997.

OLIVEIRA, D. S. et al. Avaliação do risco cardiovascular segundo os critérios de Framingham em pacientes com diabetes tipo 2. Arquivo Brasileiro de Endocrinologia e Metabolismo, v. 51, n. 2, p. 268-274, 2007.

PERDIGÃO, C. Diabetes e doença cardiovascular. Sociedade Portuguesa de Cardiologia, 2009. Disponível em: <http://www.spc.pt/DL/RFR/artigos/198.pdf>. Acesso em: 10 set. 2015.

POZZAN, R. et al. Dislipidemia, Síndrome Metabólica e Doença Cardiovascular. Revista Sociedade de Cardiologia do Estado do Rio de Janeiro, n.17, v. 2, p. 97-104, 2004. 